\title{
Based on RFID and NFC Technology Retail Chain Supermarket Mobile Checkout Mode Research
}

\author{
Fugui Ruan \\ School of Computer \& Information Science \\ Chongqing Normal University \\ Chongqing, China \\ ruanfg168@163.com
}

\author{
Daijiang Chen \\ School of Computer \& Information Science \\ Chongqing Normal University \\ Chongqing, China \\ 335421766@qq.com
}

\begin{abstract}
Radio Frequency Identification (RFID) is a non-contact automatic identification technology. NFC technology is an extension of RFID technology. This paper analyzes the application status of RFID and NFC technology and introduces the working principle of RFID, NFC technology and EPC system. Based on RFID and NFC technology, the paper proposes a mobile payment way in retail chain supermarket that can realize fast checkout.
\end{abstract}

Keywords-RFID; Retail Chain Supermarket; EPC Network; NFC; Mobile Checkout

\section{INTRODUCTION}

At present, most of the retail chain supermarket products identification is still based on the bar code scanning technology. This identification has some disadvantages, such as it is not convenient to manage goods shelves, likely to cause replenishing not on time, the customer can't quickly query the products detailed information. The biggest disadvantage is that the cashier must scan product bar codes one by one in order to obtain the product price. It is obvious that the way of product identification can not satisfy the demand of fast checkout. If customers can use NFC smart phone realizing checkout on the shopping trolleys, it will greatly shorten the time of checkout.

\section{RESEARCH and APPLICATION of RFID and} NFC

The traditional Enterprise application integration (EAI) is suitable only for integrating different application virtual information. Based on the RFID technology, the EAI frame can real-time processes production data and dynamically tracks and controls production process [1]. Every goods is affixed a RFID tags before it is transported, then the RFID tags are scanned via reader in order to getting goods transport information at every passing node, and do that the losing goods can be found as soon as possible and find them out via EPC Network [2]. Via RFID tags and wireless sensors that are equipped in the freight wagon, the temperature and humidity can be monitored continuously. This should be a result that the rotten products can be removed on time and the remedial measures are taken to prevent the products going worse [3].

At bus stop or subway station, passengers can use NFC smart phone purchasing tickets instead of the bus card [4]. If put the NFC tags in the scenic tourist map, which have stored detail information of scenic spots, the tourists can get scenic introduction video, resort feature pictures, historical allusions and so on via NFC smart phone [5]. 


\section{RFID WORKING PRINCIPLE, EPC NETWORK and NFC TECHNOLOGY}

RFID (Radio Frequency Identification) is a automatic identification technology, which utilizes the spatial coupling of electromagnetic signal to achieve non-contact transmission of the object information. A typical RFID system consists of RFID tags, built-in antennas and RFID readers. The general workflow of RFID system: When The RFID tag enters the scope of the reader antenna working, the RFID tag is activated, and then the RFID tag transmits the stored information to the reader via built-in antenna, the reader via built-in antenna receives the information and sends the information to management system for further processing.

EPC Network defines an automatic real-time identification and the supply chain information sharing platform based on the RFID radio frequency identification technology. EPC Network mainly composes of EPC coding, RFID tags, RFID readers, Savant, Object Name Service (ONS), Physical Markup Language (PML), Information Service (IS) server and Internet [6]. Workflow: The reader reads EPC code information stored in a RFID tag and sends EPC it to Savant for processing, after that the local IS server begin to find the product specific information according to the savant inquire and return it to the savant. But if the information is not fount, the Local IS server sends a request to a ONS server on the internet and the ONS server returns the IP address of the remote IS server that stores the product specific information, then the savant accesses the remote IS server to obtain the product specific information and stores it in the local IS server for next inquiry.

Near Field Communication (NFC) is developed from RFID technology and has some advantages, such as the strong confidentiality, the strong anti-interference and the identification of a short time. The differences between RFID and NFC are that the ten centimeter transmission distance, the bi-directional connection and identification features, and the high security.

At present, most of NFC mobile payment applications belong to micro payment. The applications mainly include subway station purchasing ticket, movie ticket voucher, fast-food payment, payment of a vending machine, etc. On the basis of RFID application to retail chain supermarket and combining with NFC function of smart mobile, the paper proposes a fast moving retail chain supermarket checkout.

\section{RETAIL CHAIN SUPERMARKET MOBILE CHECKOUT SYSTEM}

The mode of the retail chain supermarket mobile checkout is shown in Fig. 1.

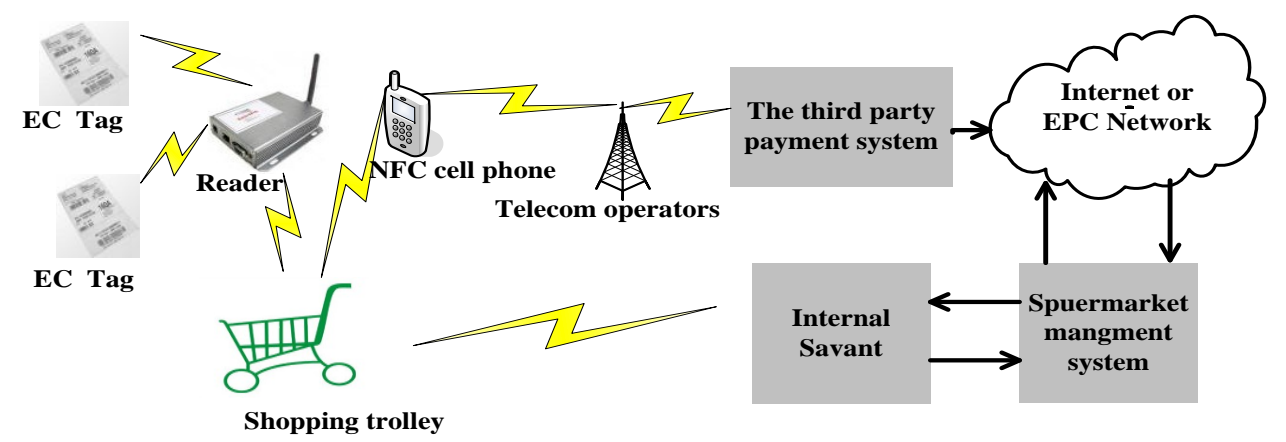

Figure 1 Mobile checkout mode 
In the whole system NFC mobile phone and the third-party payment hold an important position. The NFC mobile phone stores the payment certificate, security keys, and payment application and provides the non-contact communication interface. The third-party certificate, manages the information of user accounts, and provides the retail chain supermarkets payment interference and data certificate.

Retail chain supermarket mobile checkout trolley function module is shown in figure 2.

payment service providers mainly issue payment

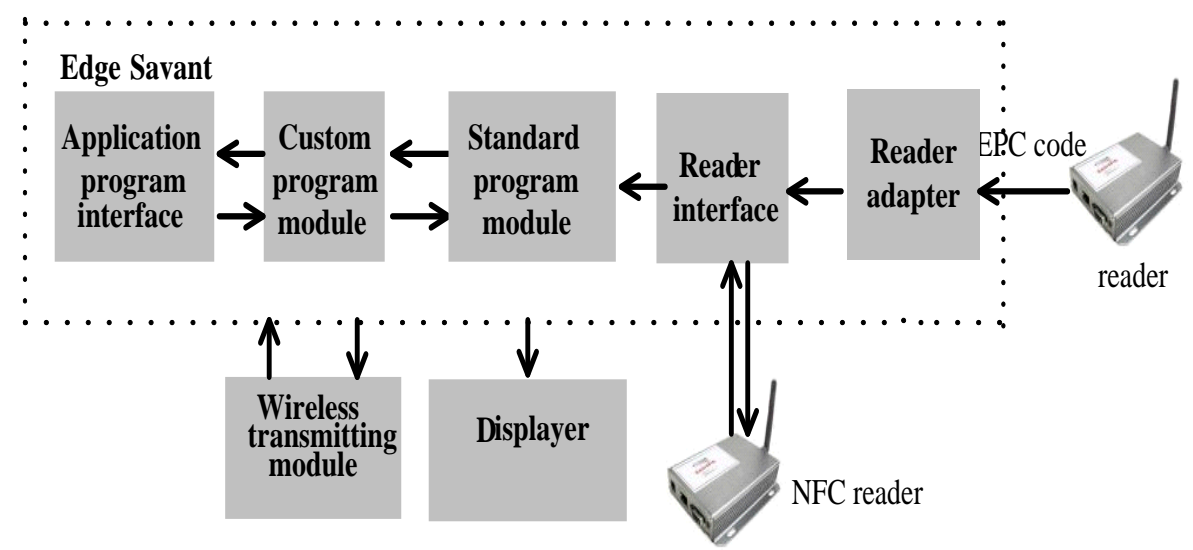

Figure 2 Shopping trolley module

From the EPC Network structure point of view, the shopping trolley is equivalent to edge savant, preliminarily processes events flow and information that is obtained by readers from tags. The RFID and NFC reader combine reader interface via reader adapter. The reader adapter transforms RFID tags information into a unified format and then transmits it to reader interface. The reader interface communicates with standard program modules. The standard program module event filter is the mainly program module defined by user, which is used to realize real-time calculating the total price of all products in a shopping trolley. User-defined program module also realizes anther function is that once the shopping trolley recognize NFC smart mobile it immediately precedes to checkout status. The application program interface is used to realize connecting the internal savant and the retail chain supermarket management system. The display is used to display the list of products information, advertisements promotion information and navigation information.

The data flow of customer use NFC smart phone realizing checkout in retail chain supermarket as shown in figure 3 . 


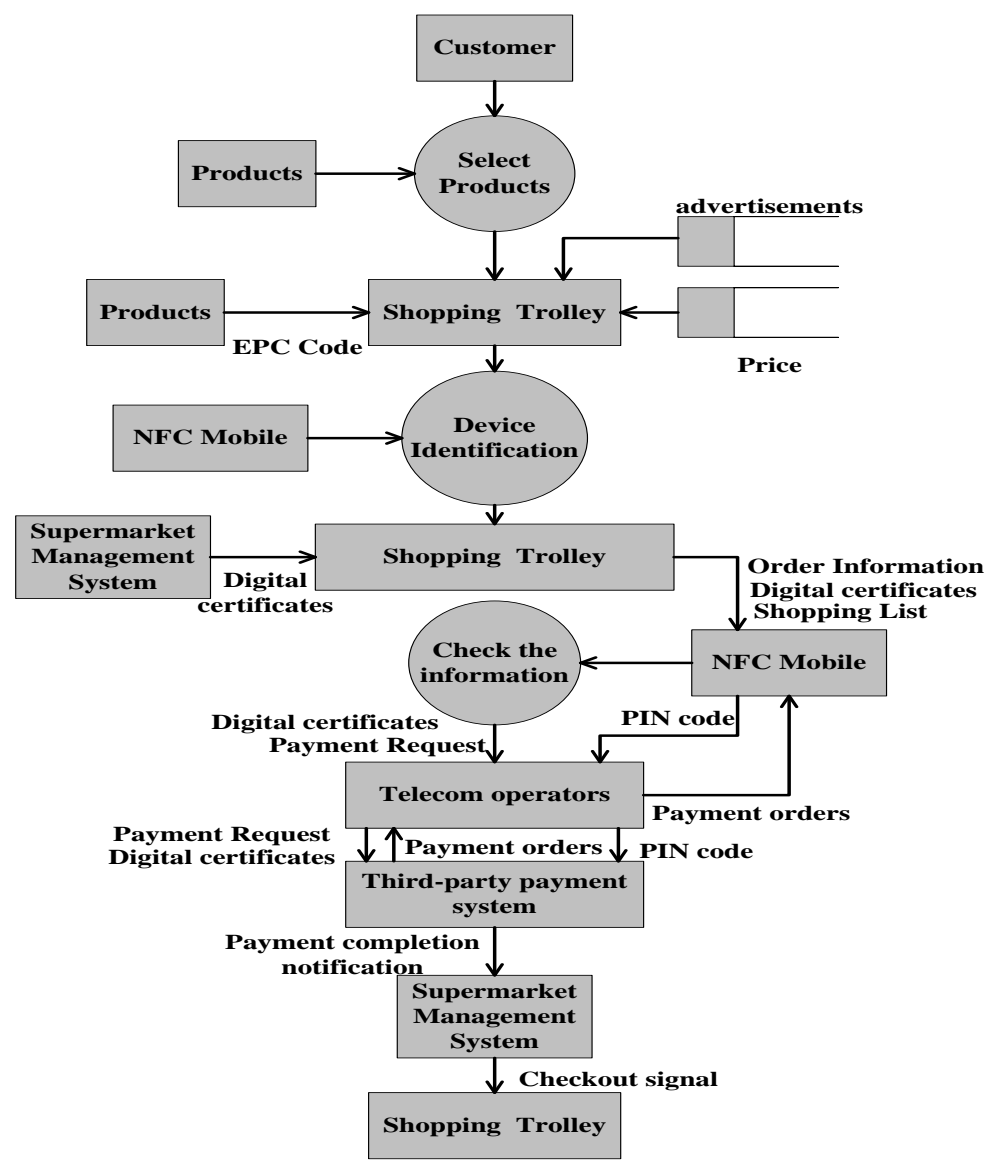

Figure 3 Checkout data flow

1) When customer pushes a shopping trolley and browse products among goods shelves, readers located in goods shelves transmit products EPC code information to the shopping trolley. The shopping trolley communicates with the retail chain supermarket management system and connects EPC Network in order to obtain products details. The shopping trolley automatically calculates the total product price.

2) The NFC reader on the shopping trolley identifies NFC smart phone of customer, the shopping trolley generate order and transmits the order, the shopping list and the digital certificate to NFC mobile application program of customer via NFC reader.

3) Customer checks the price and the shopping list. The NFC mobile automatically transmits the digital certificate of the retail chain supermarket to the Third-party payment system for authenticating. The third-part payment system returns on the retail chain supermarket digital certificate authentication information. Customer enters PIN code for identification after customer confirms the order information and the digital certification correctness.

4) The NFC mobile transmits payment request to the third-part payment system after customer identification via mobile communication network. The third-part payment system versifies the submit information from customer, and confirms the validity of payment inquire according to customer account.

5) The third-part payment system generates payment order and sends it to customer NFC mobile after via 
mobile communication network after that the payment request submit is confirmed by the third-part payment system.

6) Customer checks payment order and confirms payment. The third-part payment system sends payment accomplished information to the retail chain supermarket.

7) The retail chain supermarket sends payment completed signal to the shopping trolley.

The mobile checkout function of NFC smart phone composes of two parts in hardware: NFC analog front-end and security unit. The security unit can be divided into the SIM card and SD card depending on the different operators and this paper selects the SIM card as the security unit. From the point of view of the technology compatibility and technology, NFC+SWP solution have the most advantage, which is one kind of connection way between NFC chip and SIM card. Single Wire Protocol (SWP) is proposed by the Gemalto Company, which stipulates that NFC chip connect SIM card via C6 pin of the SIM card. The connection solution can still work in the case of power outages [7]. The NFC chips realizes no-contact function and transmits payment data to the SIM card, then the payment data is exchanged between the NFC smart phone and the third-part payment system via mobile Network.

\section{THE REATAIL CHAIN SUPERMARKET MOBILE CHECKOUT FEATURES}

1) The combination of remote payment and face-to-face payment

The remote payment is realized by deducting call charge via SMS, USSD, WAP, IVR, and Web. The proposed mobile payment forms order face-to-face, then paying via the remote third-part payment system, and can achieving a large amount payment.

2) Compatibility with existing equipments

The NFC and most present RF equipments operate in the $13.56 \mathrm{MHz}$ frequency band, such as RFID tags, readers. So it will be easy to design NFC reader, NFC tag on the basis of existing RFID tag and reader.

3) Higher security

Traditional mobile payment is implemented via SMS or IVR, etc. Information transmit in traditional mobile payment is plaintext. The proposed retail chain supermarket mobile payment can adopt NFC+SIM security module, the security has a better encryption function.

\section{CONCLUSION}

With RFID chips cost reducing and NFC smart mobile phone popularizing, the way of choosing products and payment in retail chain supermarket will completely charge. However, as supermarket checkout amount is large, security issues must be resoled. Compared with the traditional mobile payment, the security of the proposed mobile payment in retail chain supermarket has been improved, but there are still security threats, therefore, it is need to further research in mobile payment security issues.

\section{References}

[1] Ruey-Shun Chen, Mengru Arthur Tu and Jung-Sing Jwo: An RFID-based enterprise application integration framework for real-time management of dynamic manufacturing processes. Int $\mathrm{J}$ Adv Manuf Technol (2010) 50:1217-1234

[2] Jong Myoung Ko, Choonjong Kwak, Youngho Cho and Chang Ouk Kim: Adaptive product tracking in RFID-enabled large-scale supply chain. Expert Systems with Applications 38 (2011) 1583-1590

[3] Che-Fu Hsueh and Mei-Shiang Chang : A Model for Intelligent Transportation of Perishable Products. Int. J. ITS Res. (2010) $8: 36-41$

[4] Florian Resatsch: Working prototype: An NFC-based Mobile Phone Ticketing System. Gabler. 2010

[5] Francisco Borrego-Jaraba, Irene Luque Ruiz and Miguel A 'ngel Go'mez-Nieto: A NFC-based pervasive solution for city touristic surfing. Pers Ubiquit Comput (2011) 15:731-742

[6] Xian-Yi Chen and Zhi-Gang Jin: Research on Key Technology and Applications for Internet of Things. Physics Procedia 33 (2012) 561566

[7] Huang Kui, Zhuang Wenya: Based on NFC Mobile Payment Solution. Technology Application. 2010 